\title{
Collisions of solid particles with vortex rings in superfluid helium $\dagger$
}

\author{
DEMOSTHENES KIVOTIDES AND S. LOUISE WILKIN \\ Center for Risk Studies and Safety, Department of Chemical Engineering, University of \\ California, Santa Barbara, CA 93117, USA \\ (Received ?? and in revised form ??)
}

We have performed self-consistent computations of the interactions between a superfluid vortex-ring and a solid-particle for two different vortex-ring sizes and over a wide range of temperatures. In all cases, the particle and the vortex eventually separate. For temperature $T=0 \mathrm{~K}$, larger rings tend to trap the particle more effectively than smaller rings. Trying to escape the vortex, the particle follows a spiraling trajectory that could be experimentally detected. The dominant dynamical process is the excitation and propagation of Kelvin waves along the vortices. For $T>0 \mathrm{~K}$, particle-vortex collision induces particle-vibrations that are normal to the particle's direction of motion and might be experimentally detectable. In contrast to the $T=0 \mathrm{~K}$ case, smaller rings induce larger particle oscillation velocities. With increasing temperature, enhanced mutual friction damping of Kelvin waves leads to the damping of both the intensity and frequency of post-collision particle vibrations. Moreover, higher temperatures increase the relative impact of the Stokes drag force on particle motion.

\section{Introduction}

The physics of particle laden flows can be studied at various space-time scales employing appropriately designed mathematical models (see for example Crowe, Sommerfeld \& Tsuji 1998; Drew \& Passman 1998; Brennen 2005). One standard method of inquiry assumes that the particles are spherical, and that their size is much smaller than the smallest spatial scale of a corresponding pure flow with the same initial and boundary conditions, but without any particles. In turbulent flows, the latter scale could be identified with the Kolmogorov microscale of turbulence. Under these conditions, there is always a creeping flow of very small Reynolds number around the particles, which therefore could justifiably be neglected in comparison to an energetic large scale flow. A similar accompanying assumption is that the particle-field is so dilute, that hydrodynamic interactions between particles could also (most of the time) be neglected. Hence, the solution of the corresponding multi-body Stokes micro-flow problem (with either point or finite-size particles) could be avoided. When such assumptions of one-way coupling are employed, one can use analytical solutions of the flow field around an isolated sphere in a uniform low Reynolds number flow stream, in order to find closed-form expressions for the force that the fluid exerts on the particle. Since such expressions take analytically into account the developed microflow around the particle, a direct numerical computation of this flow is not necessary. This method allows an economical and adequate treatment of particle laden normal-fluid flows, whenever the aforesaid assumptions are valid.

$\dagger$ This paper is dedicated to the memory of Brian Jackson. 
Recently, a number of experiments have realized particle laden flows in liquid helium (Donnelly, Karpetis, Niemela, Sreenivasan, Vinen \& White 2002; Celik \& VanSciver 2002; Zhang, Celik \& VanSciver 2004; Zhang \& VanSciver 2005; Bewley, Lathrop \& Sreenivasan 2006). The latter are two component physical systems where a normal-fluid interacts via mutual friction forces with a superfluid (Donnelly 1991; Barenghi, Donnelly \& Vinen 2001; Vinen \& Niemela 2002; Finne, Eltsov, Hanninen, Kopnin, Kopu, Krusius, Tsubota \& Volovik 2006). At present, there are no methods for the direct, unambiguous measurement of the local flow velocity in any of the two fluids. The idea behind these experiments is to release micron-sized particles in liquid helium, and by measuring their velocity (via the Particle Image Velocimetry (PIV) technique) to make inferences about the velocity of the two constituent fluids. A first theoretical analysis of such flows was offered by Poole et al. (2005). This analysis was based on assumptions very similar to those mentioned above for the treatment of normal multiphase flow. Unfortunately, such an approach encounters severe difficulties when applied to superfluids. In particular, the latter are characterized by quantized, potential vortices of nanometer-sized cores which correspond to spatial flow scales that are orders of magnitude smaller than the micronsized diameters of the inserted particles. Because of these, Kivotides, Barenghi \& Sergeev $(2005,2006 b)$ had to confine themselves to dilute particle-fields in order to show that when the particles are located many particle diameters away from superfluid vortices, they are forced by the viscous drag to trace the normal-fluid and thus PIV methods could successfully be employed to measure the normal-fluid velocity. Hence, multiphase superfluids require new, more powerful methods for their mathematical description. Recently, Kivotides, Barenghi \& Sergeev $(2006 a, 2007 b, 2008)$ have developed methods for the treatment of particle vortex interactions from first principles, without any scale separation or one-way coupling hypotheses. In this article, we first formulate the mathematical problem that corresponds to the collision of a spherical particle with a superfluid vortex ring, and then (using the recently developed methods), we solve this problem for various particle ring configurations of importance to superfluids.

\section{Mathematical model}

We are concerned with the collision of a superfluid vortex ring of radius $R$ with a solid, spherical particle of radius $a$. In general, let $\mathbf{X}(\ell, \mathrm{t})$ denote a superfluid vortex link $\mathcal{L}$ where $\ell$ is the arclength parametrization along the vortex loops and $t$ is time. The dynamics of $\mathbf{X}(\ell, \mathrm{t})$ are described by the following integrodifferential equation:

$$
\frac{\partial \mathbf{X}}{\partial t}=\mathbf{V}^{s}+\mathbf{V}^{b}+\mathbf{V}^{\phi}+\mathbf{V}^{f} .
$$

The first contribution to the right hand side is the superfluid velocity $\mathbf{V}^{s}$ that is given by the Biot-Savart integral:

$$
\mathbf{V}^{s}(\mathbf{x})=-\frac{\kappa}{4 \pi} \int_{\mathcal{L}} \mathrm{d} \ell \frac{\mathbf{X}^{\prime} \times(\mathbf{X}-\mathbf{x})}{|\mathbf{X}-\mathbf{x}|^{3}},
$$

where $\mathbf{X}^{\prime} \equiv \partial \mathbf{X} / \partial \ell$ is the unit tangent vector (indicating the direction of the singular superfluid vorticity) and $\kappa$ is the quantum of circulation.

The particle acts like an obstacle that deforms the vortex induced flow, since the latter can not penetrate the spherical boundary of the former. This (particle-induced) flow deformation advects the vortices and this effect is denoted by the term $\mathbf{V}^{b}$. The computation of $\mathbf{V}^{b}$ is discussed by Schwarz (1974) and Kivotides et al. (2006a). The latter 
reference provides detailed, instability-free formulae for the computation of $\mathbf{V}^{b}$ that we have employed in this work.

The third contribution $\mathbf{V}^{\phi}$ is the potential flow field induced by the motion of a spherical particle with velocity $\mathbf{V}^{p}$ as it moves in a stationary, unbounded, inviscid fluid. This flow field is given by the expression (Drew \& Passman 1998):

$$
\begin{aligned}
\mathbf{V}^{\phi}(\mathbf{x} \mid \mathbf{z}) & =-0.5\left(\frac{a}{r}\right)^{3} \mathbf{V}^{p}(\mathbf{z}) \cdot\left(\mathbf{I}-3 \frac{\mathbf{x}^{\prime} \mathbf{x}^{\prime}}{r^{2}}\right), \\
V_{j}^{\phi}(\mathbf{x} \mid \mathbf{z}) & =-0.5\left(\frac{a}{r}\right)^{3} V_{i}^{p}(\mathbf{z})\left(\delta_{\mathbf{i j}}-3 \frac{\mathbf{x}_{\mathbf{i}}^{\prime} \mathbf{x}_{\mathbf{j}}^{\prime}}{r^{2}}\right),
\end{aligned}
$$

where $\mathbf{V}^{\phi}(\mathbf{x} \mid \mathbf{z})$ is the velocity of the fluid at $\mathbf{x}$ caused by a sphere of radius $a$ whose centre is located at $\mathbf{z}, \mathbf{I}$ is the $3 \times 3$ unit matrix whose elements are the Kronecker delta symbols $\delta_{i j}(i, j=1,2,3), \mathbf{x}^{\prime}=\mathbf{x}-\mathbf{z}, r=|\mathbf{x}-\mathbf{z}|$, and a summation over index $i$ is implied in the component form of the equation.

Since in this work we compute evolutions for both pure $(T=0 \mathrm{~K}$, where $T$ is the temperature) superfluid helium and superfluid helium at nonzero temperature, we need to take into account the effects of the mutual friction force on the vortices. These effects correspond to the fourth term $\mathbf{V}^{f}$ that is given by the formula:

$$
\begin{aligned}
\mathbf{V}^{f}=h_{\star \star}\left(\mathbf{V}^{s}+\mathbf{V}^{b}+\mathbf{V}^{\phi}\right) & +h_{\star} \mathbf{X}^{\prime} \times\left(\mathbf{V}^{n}-\left(\mathbf{V}^{s}+\mathbf{V}^{b}+\mathbf{V}^{\phi}\right)\right) \\
& +h_{\star \star} \mathbf{X}^{\prime} \times\left(\mathbf{X}^{\prime} \times \mathbf{V}^{n}\right),
\end{aligned}
$$

where $\mathbf{V}^{n}$ is the kinematically prescribed normal-fluid velocity, and $h_{\star}=d_{\star \star} /\left(d_{\star \star}^{2}+(1-\right.$ $\left.\left.d_{\star}\right)^{2}\right), h_{\star \star}=\left(d_{\star}-d_{\star \star}^{2}-d_{\star}^{2}\right) /\left(d_{\star \star}^{2}+\left(1-d_{\star}\right)^{2}\right)$ are dimensionless numbers given in terms of the (also dimensionless) mutual friction coefficients $d_{\star}$ and $d_{\star \star}$. To compute the latter coefficients, one starts from the Hall-Vinen coefficients and following Barenghi, Donnelly \& Vinen (1983) calculates the transverse and longitudinal drag coefficients $D_{\star}^{\prime}$ and $D_{\star \star}$. Subsequently, one computes $D_{\star}=D_{\star}^{\prime}-\rho_{n} \kappa$, and finally $d_{\star}=D_{\star} /\left(\rho_{s} \kappa\right), d_{\star \star}=D_{\star \star} /\left(\rho_{s} \kappa\right)$. The particle dynamics is described by the following equation:

$$
m_{e} \frac{\mathrm{d} \mathbf{V}^{\mathbf{p}}}{\mathrm{d} t}=6 \pi a \mu_{n}\left(\mathbf{V}^{n}-\mathbf{V}^{p}\right)+2 \pi \rho_{s} a^{3} \frac{\partial \mathbf{V}^{s}(\mathbf{z}, t)}{\partial t}+\frac{1}{2} \rho_{s} \int_{S} \mathrm{~d} S\left(\mathbf{V}^{s}+\mathbf{V}^{b}\right)^{2} \hat{\mathbf{n}},
$$

where $m_{e}$ is the effective mass of the particle $m_{e}=m+(2 / 3) \pi\left(\rho_{s}+\rho_{n}\right) a^{3}, m$ is the particle mass, $\mu_{n}$ is the dynamic viscosity of the normal-fluid, $\rho_{s}$ and $\rho_{n}$ are the superfluid mass density and normal-fluid mass density respectively, and $\mathbf{V}^{s}(\mathbf{z}, t)$ is the vortex-induced velocity at the particle centre. We will only consider neutrally buoyant particles here, thus the particle mass density $\rho_{p}$ is equal to the sum of the superfluid and normal-fluid mass densities. The last term is a surface integral with $\hat{\mathbf{n}}$ being the outward unit radial vector field on the spherical particle surface. It is convenient to write the right hand side of equation (2.5) as $\mathbf{f}=\mathbf{f}_{\mathbf{d}}+\mathbf{f}_{\mathbf{l}}+\mathbf{f}_{\mathbf{b}}$; this decomposes the total force $\mathbf{f}$ into viscous drag, $\mathbf{f}_{\mathrm{d}}$, local, $\mathbf{f}_{\mathbf{l}}$, and boundary force, $\mathbf{f}_{\mathrm{b}}$.

Note that, although the equation of motion for the vortices is exact for both $T=0 \mathrm{~K}$ and finite temperature liquid helium, the same is not true for the particle equation of motion. The latter is exact only at $T=0 \mathrm{~K}$ in which case it reduces to the important Schwarz's solid-particle equation of motion Schwarz (1974). The equation employed here takes heuristically into account thermal effects via a viscous drag force known as Stokes force (the first term on the right hand side), and an added mass term on the left hand side that is nonzero when the particle accelerates inside the normal-fluid. An exact treatment of normal-fluid effects could be made by solving the normal-fluid Navier-Stokes equation around a suspension of solid-particles, and taking into account the normal-fluid effect on 
the particles by replacing the Stokes-drag force in the particle equation of motion by the force

$$
f_{i}^{n}=\int_{S} \sigma_{i j} \hat{n}_{j} \mathrm{~d} S
$$

where

$$
\sigma_{i j}=-p \delta_{i j}+\mu_{n}\left(\partial V_{i}^{n} / \partial x_{j}+\partial V_{j}^{n} / \partial x_{i}\right)
$$

is the normal-fluid stress tensor, $p$ is the normal-fluid pressure, repeated indices are summed, and the integral is performed over the particle's surface. This treatment is not within the capabilities of already developed methods and it is a desirable future development.

All computations were performed in an unbounded fluid domain. This was also assumed in Schwarz's derivations of the formulae for $\mathbf{V}^{b}$, as well as of the particle equation of motion at $T=0 \mathrm{~K}$. Note, that although the $\mathbf{V}^{b}$ formulae are valid also for vortex loops in periodic domains, the $\mathbf{V}^{\phi}$ formula assumes an isolated particle and does not comply with periodic boundary conditions. The introduction of "image" particles and their effects on the vortices via the corresponding $\mathbf{V}^{\phi}$ and $\mathbf{V}^{b}$ fields are thus necessary in the latter case. Finally, in all computations of superfluid helium at nonzero temperature reported here, we have prescribed the normal flow kinematically, and we have set $\mathbf{V}^{n}=\mathbf{0}$.

\section{Mathematical methods}

A detailed discussion of the numerical and computational methods is given in Kivotides et al. (2008). For completeness of exposition, we provide here a summary. The coupled system of integro-differential equations (2.1) and (2.5) is solved using a third order accurate, low storage Runge-Kutta (RK) method. This approach requires the discretization of the Biot-Savart integral in the fashion of Riemann sums. The desingularization of the kernel is achieved by splitting it into local and nonlocal parts. The local contribution is handled following a method by Schwarz (1985). The nonlocal contribution is computed by employing a Gaussian kernel in order to smooth the singular superfluid vorticity. The numerical analysis of this method has been described by Winckelmans \& Leonard (1993). The method introduces a cut-off scale in the Biot-Savart formula which, in all results reported here, is equal to 1.2 times the discretization length along the vortices. Two vortices reconnect instantaneously when they approach each other at a distance less than the discretization length along their contours. In this way, the small scale vortex dynamics during reconnection as described by deWaele \& Aarts (1994) are not taken into account. This is consistent with the unavailability of very fine spatial resolution along the vortices as dictated by computational complexity constraints. It is also consistent with the aforesaid small distance smoothing of the superfluid singular vorticity according to the Winckelmans-Leonard method.

The method for the computation of $\mathbf{V}^{b}$ was introduced in Schwarz (1985) and the particular computational formulae are available from Kivotides et al. (2006a). It is based upon an analytical solution in terms of Legendre functions series of the effect of the spherical particle boundary on a linear vortex segment. The treatment of particle-vortex collisions involves a number of important issues that were resolved in Kivotides et al. (2008). It builds upon earlier works of Schwarz (1985) and Tsubota \& Maekawa (1993) dealing with vortex collisions with stationary particles, and it extends the latter works in the realm of self-consistent particle vortex interactions. Kivotides et al. (2006a) have shown 
that when a vortex ring propagates in the vicinity of a solid-particle, a vortex instability develops and an attachement of the particle to the vortex follows. At first sight, such an attachement leads to discontinuous vortex lines and therefore the Biot-Savart line integral becomes ill-defined. Our method tackles this problem by continuing the attached vortex inside the particle (i.e. by preserving the continuity of the line vortices), and by taking into account in a self-consistent way the effect of the added vortex segments on the dynamics of the vortices outside the particle. These effects include not only Biot-Savart effects of the inner-particle vortex segments on the outer vortices, but also an extra velocity contribution to the outer vortex dynamics which ensures that the addition of inner vortices complies with the no flow condition normal to the particle's surface. Kivotides et al. (2008) provides detailed discussions of the various numerical and computational techniques involved, as well as evidence about the accuracy and efficiency of our approach.

\section{Results}

We shall apply our methods to ${ }^{4} \mathrm{He}$. Since superfluidity in ${ }^{4} \mathrm{He}$ exists over a temperature range starting from $T=0 \mathrm{~K}$ where only the superfluid is present, to a temperature (infinitesimally) below that of the Bose-Einstein condensation where the superfluid component is negligible, a thorough investigation of every superfluid problem ought to include a temperature space parameter search. In this work, we compute particle-vortexring interactions at four different temperatures: $T=0 \mathrm{~K}$ (where $\rho_{s}=0.145 \mathrm{~g} \mathrm{~cm}^{-3}$ and $\rho_{n}=0$ ), $T=1.3 \mathrm{~K}$ (where $\rho_{s}=0.1386 \mathrm{~g} \mathrm{~cm}^{-3}, \rho_{n}=0.00652 \mathrm{~g} \mathrm{~cm}^{-3}$, and $\mu_{n}=$ $\left.15.2 \times 10^{-6} \mathrm{~g} \mathrm{~cm}^{-1} \mathrm{~s}^{-1}\right), T=1.95 \mathrm{~K}$ (where $\rho_{s}=0.07542 \mathrm{~g} \mathrm{~cm}^{-3}, \rho_{n}=0.07012 \mathrm{~g} \mathrm{~cm}^{-3}$, and $\left.\mu_{n}=14.1435 \times 10^{-6} \mathrm{~g} \mathrm{~cm}^{-1} \mathrm{~s}^{-1}\right)$, and $T=2.171 \mathrm{~K}\left(\right.$ where $\rho_{s}=0.00652 \mathrm{~g} \mathrm{~cm}^{-3}$, $\rho_{n}=0.13955 \mathrm{~g} \mathrm{~cm}^{-3}$, and $\left.\left.\mu_{n}=25.40145 \times 10^{-6} \mathrm{~g} \mathrm{~cm}^{-1} \mathrm{~s}^{-1}\right)\right)$. For the two lowest temperatures, we used two different ring radii: $R=0.00025 \mathrm{~cm}$ and $R=0.00125 \mathrm{~cm}$, while for the two larger temperatures, we have studied only the $R=0.00125 \mathrm{~cm}$ case. This is because for the larger temperatures, the $R=0.00025 \mathrm{~cm}$ rings were shrinking so fast that (for the initial particle/ring distance mentioned above) collisions were not occurring. In other words, the particle and the ring did not reach a close enough proximity for the latter to become unstable and attach to the spherical surface as was shown in Kivotides et al. $(2006 a)$. Although we have performed computations with zero initial particle velocities, we have also performed a search in velocity space for two cases, i.e. $T=1.95 \mathrm{~K}$ and $T=2.171 \mathrm{~K}$. The particle diameter $2 a=2 \times 10^{-4} \mathrm{~cm}$ was chosen in agreement with particle sizes used in experiments. All computations reported here were done with this particle size. Moreover, in all cases, the initial particle-vortex distance was $1.5 a$. The vortex velocity is directed towards the positive $y$-axis, and the $z$-axis is along the vertical direction.

The accuracy of all results presented here was continuously monitored throughout all computations. Since the scope of our method is to enforce the zero radial velocity boundary conditions on the surface of the particle, we have devised an appropriate accuracy criterion. In particular, if the boundary condition was enforced exactly, at all points on the particle's surface the total velocity vector would form a 90 degrees angle with the radial direction vector. We allow small computational deviations from this exact condition only up to a given tolerance from 0.01 to 0.1 degrees. This is achieved with a modest number of terms in the Legendre expansion, of the order of 100 . We have also verified the accuracy of the purely vortex dynamics algorithms. It is important that the latter enforce the conservation of energy during vortex ring propagation at zero temperature. In practice, this means that the variations of the ring's diameter and planarity 


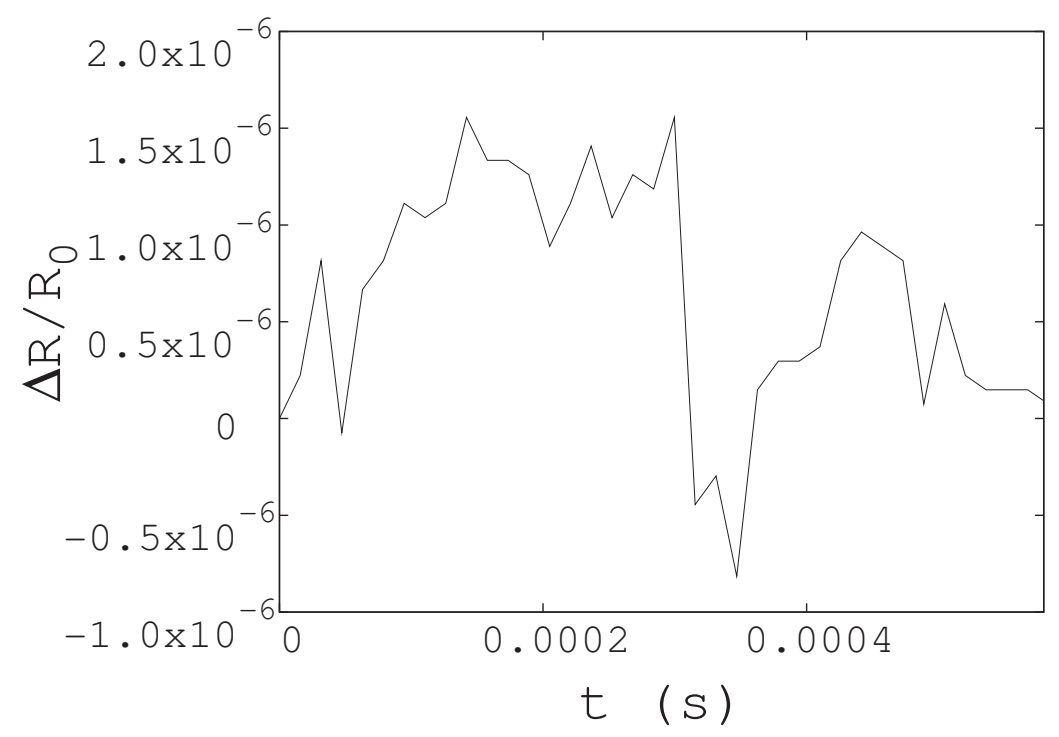

FiguRE 1. Relative error in the vortex-ring radius data $\Delta R / R_{0}$ versus time. The ring has radius $R_{0}=0.00025 \mathrm{~cm}$ and propagates at $T=0 \mathrm{~K}$. There were approximately $3.7 \times 10^{4}$ time-steps taken within this time interval.

during its motion ought to be negligible. Moreover, these must be true for the actual ring diameters and computational time-steps and discretization-lengths employed in the particle-vortex computations. Figure 1 shows the relative error $\Delta R / R_{0}$ in the ring's radius data when a ring of radius $R_{0}=0.00025 \mathrm{~cm}$ propagates at $T=0 \mathrm{~K}$ over a period of time $\Delta t=0.0006 \mathrm{~s}$. There were approximately $3.7 \times 10^{4}$ time-steps taken within this time interval. The computational specifications are idendical to the corresponding case in the actual results. Evidently, $\Delta R / R_{0}$ is of order $10^{-6}$. Morevover the maximum deviation from exact planarity (the numerical "width" of the ring) is $2 \times 10^{-8} \mathrm{~cm}$. The particle radius represents a basic length scale that must be well resolved in order to capture the relevant physics which, as the results indicate, are determined by the formation upon particle-vortex collision of a Kelvin wave system along the vortex. By choosing a grid size 12 times smaller than the particle diameter, we ensure that particle-Kelvin-wave interactions at the particle scale are well captured. This resolution requirement is met by a discretization length $\delta \ell \approx 1.56 \times 10^{-5} \mathrm{~cm}$. The time step $\delta t$ must not allow the fastest Kelvin wave in the system to propagate by more than one $\delta \ell$ per time step, and must not exceed $0.25 \tau$. Here, the Stokes time $\tau$ is given by the formula $\tau=a^{2} \rho_{p} / 3 \mu_{n}$, where $\rho_{p}$ is the mass density of the particle and $\mu_{n}$ is the dynamic viscosity of the normal-fluid. This time is the characteristic time that viscous forces need in order to damp particle motion in the absence of vortex effects. The purpose of the prefactor 0.25 is to ensure that the viscous damping process is adequately resolved. For particle diameter $2 a=2 \times 10^{-4} \mathrm{~cm}$, the three temperature cases $T=1.3 \mathrm{~K}, T=1.95 \mathrm{~K}$ and $T=2.171 \mathrm{~K}$ correspond to $\tau=3.1935 \times 10^{-5} \mathrm{~s}, \tau=3.4301 \times 10^{-5} \mathrm{~s}$, and $\tau=1.9170 \times 10^{-5} \mathrm{~s}$ respectively. The typical time step is $\delta t \approx 0.15 \times 10^{-7} \mathrm{~s}$.

Finally, in discussing the solutions, we will refer to the various contributions to the total force acting on the particle. These are the boundary induced force

$$
\mathbf{f}_{\mathbf{b}}=\frac{1}{2} \rho_{s} \int_{S} \mathrm{~d} S\left(\mathbf{V}^{s}+\mathbf{V}^{b}\right)^{2} \hat{\mathbf{n}},
$$




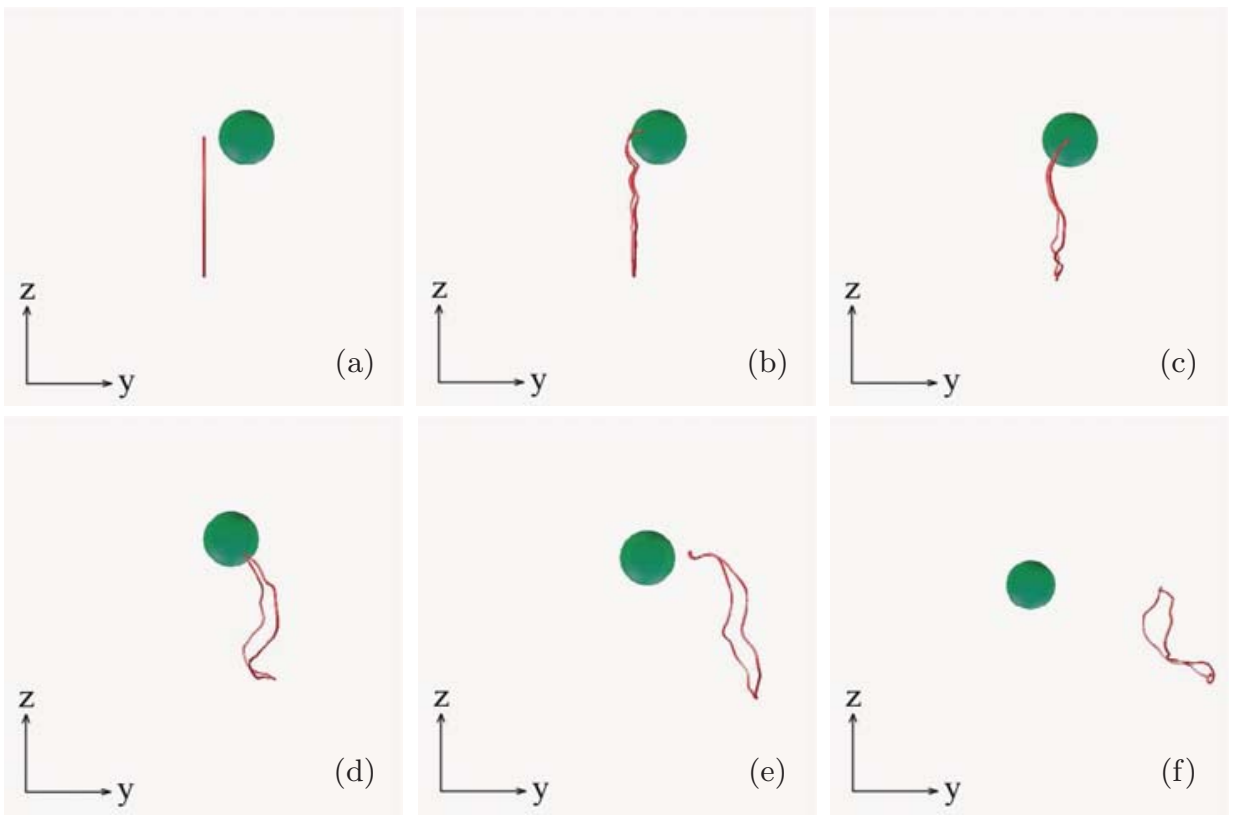

Figure 2. (Colour online.) Particle-vortex collision for initial particle speed $V=0 \mathrm{~cm} \mathrm{~s}^{-1}$, at $T=0 \mathrm{~K}$. The vortex-ring has radius $R=0.00025 \mathrm{~cm}$ and moves to the right with initial velocity $3.3465 \mathrm{~cm} \mathrm{~s}^{-1}$. The initial vortex-ring plane is normal to the figure. Time increases from the upper-left graph towards the lower-right graph as follows: (a) $t_{1}=0 \mathrm{~s}$, (b) $t_{2}=0.1892 \times 10^{-6} \mathrm{~s}$, (c) $t_{3}=0.3154 \times 10^{-6} \mathrm{~s}$, (d) $t_{4}=0.6940 \times 10^{-5} \mathrm{~s}$, (e) $t_{5}=0.1640 \times 10^{-4} \mathrm{~s}$, (f) $t_{6}=0.2208 \times 10^{-4} \mathrm{~s}$. As the vortex attaches to the particle, a system of vortex waves develops. The vortex wanders for sometime at the back side of the particle, before detaching from it.

the damping force

$$
\mathbf{f}_{\mathbf{d}}=6 \pi a \mu_{n}\left(\mathbf{V}^{n}-\mathbf{V}^{p}\right)
$$

and the local force

$$
\mathbf{f}_{\mathbf{l}}=2 \pi \rho_{s} a^{3} \partial \mathbf{V}^{s}(\mathbf{z}, t) / \partial t .
$$

\subsection{Temperature $T=0 \mathrm{~K}$}

The results for the smallest ring case are shown in figure 2. Figure 2(a) depicts the initial configuration. The ring is set on a collision course with the particle's center. Figure 2(b) shows that as the ring attaches to the particle, it develops Kelvin waves conceptually similar to the Kelvin wave cascade in pure superfluid turbulence (Kivotides et al. 2001; Vinen et al. 2003; Nazarenko \& West 2003; Kozik \& Svistunov 2004). In figure 2(c) the vortex has propagated half way through the particle's diameter before moving lower and towards the back side of the sphere where it wanders for some time, shaking the particle as it moves along its surface [figure 2(d)], before it dettaches from it [figure 2(e)]. As vortex and particle drift apart [figure 2(f)], the particle moves essentially in a straight line. The latter is evident in the results of figure 3 (left), where all components of the particle's velocity become constant after vortex-particle detachement. Figure 3 also indicates that the forces acting on the particle while it is in contact with the vortex are orders of magnitude stronger than any distant interactions between the vortex and the particle. According to figure 3 (right), the local force dominates over the boundary induced force. This is mainly due to the Kelvin wave system which developed on the vortex during the 

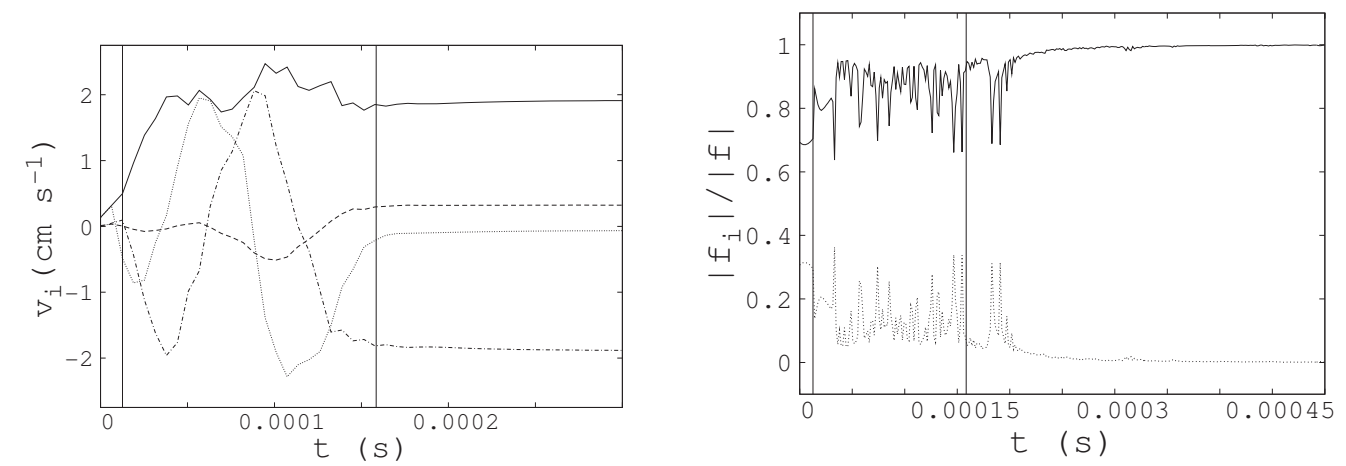

Figure 3. Particle-vortex collision for initial particle speed $V=0 \mathrm{~cm} \mathrm{~s}^{-1}$, at $T=0 \mathrm{~K}$. The particle's diameter is $2 a=2 \times 10^{-4} \mathrm{~cm}$, the ring radius is $R=0.00025 \mathrm{~cm}$, and the initial particle-vortex distance is $1.5 \mathrm{a}$. The solid vertical lines mark the times of particle-vortex collision and separation. Left: particle velocity evolution. The particle oscilates in the $y-$ (dot-dashed line) and $z-$ (dotted line) directions as indicated by the change of the velocity sign along these directions. The dynamics of the $x$-component (dashed line) are less pronounced. The solid line indicates the total velocity magnitude. After the detachement of the vortex from the particle, the latter moves essentially in a straight line. Right: partial contributions to the total force magnitude. The local force $\mathbf{f}_{\mathbf{l}}$ (solid line) dominates over the boundary force $\mathbf{f}_{\mathbf{b}}$ (dotted line).

collision. The results suggest that the introduction of particles in pure superfluid turbulent flow will have a nontrivial effect on the energetics of this flow, via the introduction of Kelvin wave cascades. The physics of these cascades would be different than the physics of the pure superfluid Kelvin wave system due to the presence of the spherical boundary. As is well known from studies of normal-fluid turbulent flows past solid surfaces, the presence of the latter alters the statistical structure of the former.

The solution for the larger ring radius $R=0.00125 \mathrm{~cm}$ appears in figure 4 . It is phenomenologically different than the previous one. As the ring attaches to the particle, the latter starts moving along circular orbits that grow in diameter with time [figure 4(b), figure 4(c)]. Figure 5 shows a detail of the $y-z$ plane projection of the particle's center trajectory after the collision shown in figure 4 . Eventually, the particle breaks free from the vortex, not because the vortex is detached from it, but because the loop reconnects with itself allowing the particle to escape having acquired a vortex 'tail' [figure 4(d)]. This could be a generic feature of interaction of particles with large vortices at ultra small temperatures, since the collision induces Kelvin waves in the vortex that in turn facilitate a self-reconnection. Why are the two solutions qualitatively different? Certainly the collision velocity plays an important role, since in the small ring case the vortex moves past the particle without allowing enough time for the latter to accelerate under the influence of the interaction forces. Indeed, the times of figure 2 differ by orders of magnitude from the times of figure 4 . Another interpretation, which also agrees with the results of Kivotides et al. (2008) at finite temperatures, is that the smaller the particle (relative to the vortex size), the more it tends to be trapped by the colliding vortex. In particular, figure 6 (left) shows the particle velocity components. It depicts that, as the particle remains attached to the vortex for longer times, its kinetic energy increases. This is suggestive of a trapping mechanism. Indeed, as in the smaller ring case, the particle would like to separate from the vortex. However, when the vortex is large, it tends to efficiently oppose this tendency by turning the particle against its direction of motion. This leads to the outwards-spiraling particle trajectories. As the forces acting on the particle change its direction, they also do work on it and increase its kinetic energy. Since 


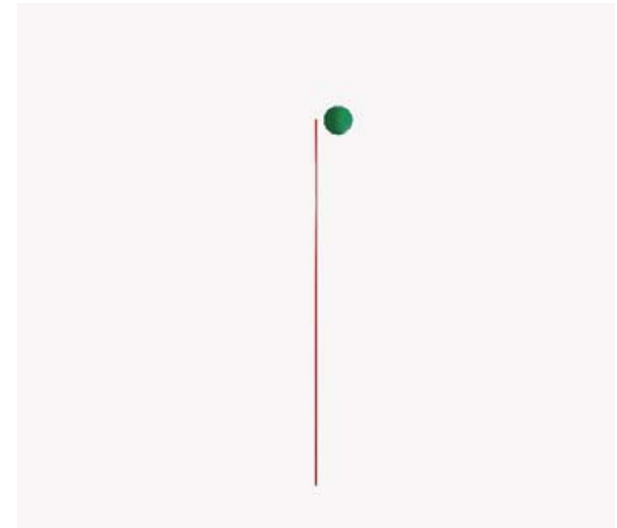

(a)

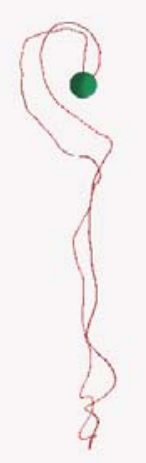

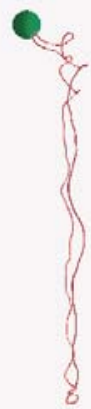

(b)

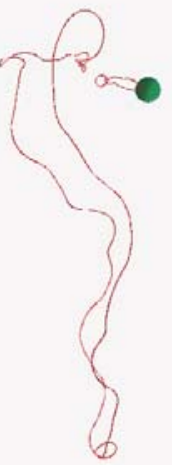

(d)

Figure 4. (Colour online.) Particle-vortex collision for initial particle speed $V=0 \mathrm{~cm} \mathrm{~s}^{-1}$, at $T=0 \mathrm{~K}$. The vortex-ring has radius $R=0.00125 \mathrm{~cm}$ and moves to the right with initial velocity $0.7714 \mathrm{~cm} \mathrm{~s}^{-1}$. The initial vortex-ring plane is normal to the figure. Time increases from the upper-left graph towards the lower-right graph as follows: (a) $t_{1}=0 \mathrm{~s}$, (b) $t_{2}=0.3407 \times 10^{-3} \mathrm{~s}$, (c) $t_{3}=0.5679 \times 10^{-3} \mathrm{~s}$, (d) $t_{4}=0.7698 \times 10^{-3} \mathrm{~s}$. The particle sprirals out from the point of initial contact, before a self-reconnection of the capturing vortex allows it to escape.

all superfluid vortices have the same circulation, larger rings correspond to larger fluid kinetic energies that act as reservoirs of available particle kinetic energy. The higher the trapping capacity of a vortex, the higher the kinetic energy that the particle acquires during its interaction with it.

Figure 7 shows the evolution of the vortex loop length (solid lines). The almost constant loop length until the onset of collision is plausible, since at this temperature there are no mutual friction effects, and, in addition, previous work (Schwarz 1985; Kivotides et al. 2006a) has shown that the vortex is being deformed by the particle only during the very late stages of its approach to it. Notice that the vortex length increases even after the particle and the vortex separate from each other. 


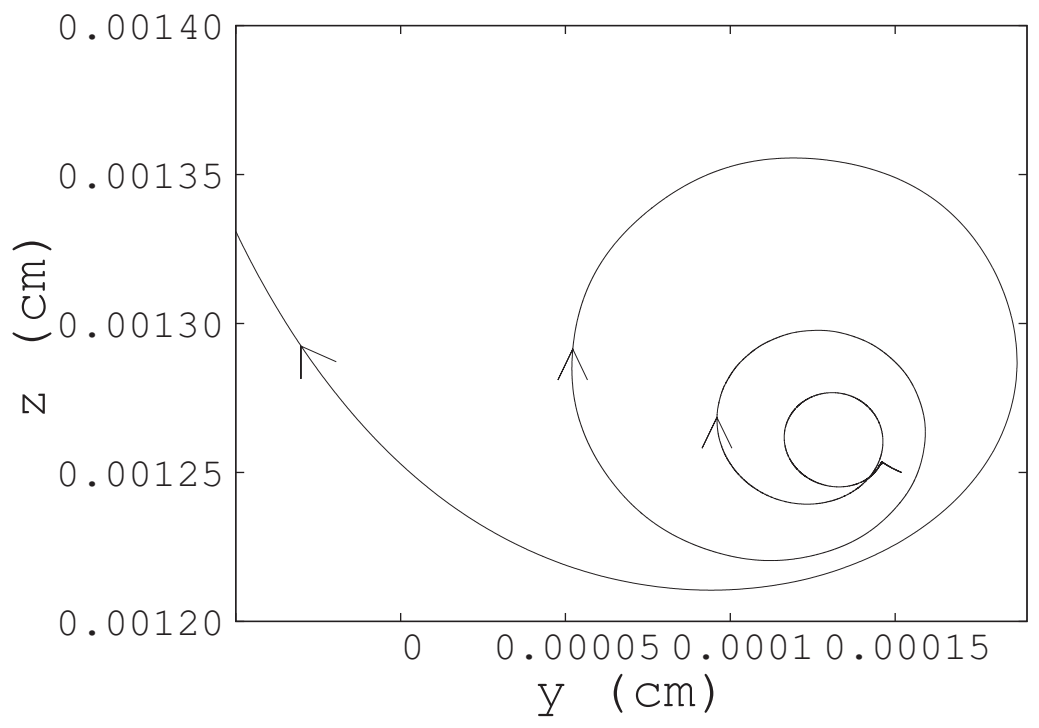

FIGURE 5. Detail of the $y-z$ plane projection of the particle's center trajectory after the collision shown in figure 4 . The arrows indicate the direction of a distinctly spiraling motion. The path shown starts at $t=0 \mathrm{~s}$ and ends at $t=0.34 \times 10^{-3} \mathrm{~s}$.
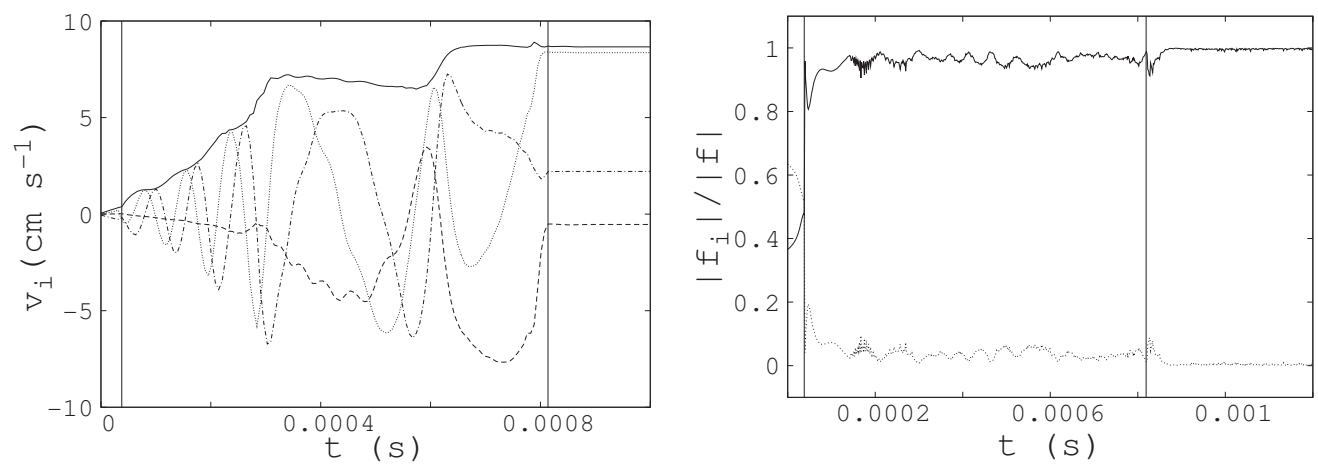

Figure 6. Particle-vortex collision for initial particle speed $V=0 \mathrm{~cm} \mathrm{~s}^{-1}$, at $T=0 \mathrm{~K}$. The particle's diameter is $2 a=2 \times 10^{-4} \mathrm{~cm}$, the ring radius is $R=0.00125 \mathrm{~cm}$, and the initial particle-vortex distance is $1.5 a$. The solid vertical lines mark the time of particle-vortex collision and separation. Left: particle velocity evolution. There are rapid variations of the $y$-component (dot-dashed line) and $z$-component (dotted line) of the velocity, that correspond to the spiraling particle motion on the $y-z$ plane. The dynamics of the $x$-component (dashed line) are less pronounced yet more interesting than in the corresponding figure 3 . The solid line indicates the total velocity magnitude. Right: partial contributions to the total force magnitude. With the exception of pre-collision times, the local force $\mathbf{f}_{\mathbf{l}}$ (solid line) dominates over the boundary force $\mathbf{f}_{\mathrm{b}}$ (dotted line). This is due to the system of Kelvin waves that develops on the vortex upon collision.

\subsection{Temperature $T=1.3 \mathrm{~K}$}

The evolution of the system's configuration for temperature $T=1.3 \mathrm{~K}$ is shown in figure 8. Although mutual friction damps the collision-induced Kelvin waves, the loop's trajectory is similar to that in the corresponding $T=0 \mathrm{~K}$ case. Figure 9 (left) shows the various components of the particle velocity, and figure 9 (right) shows the partial contributions to the total force acting on the particle. The local and boundary forces dominate 

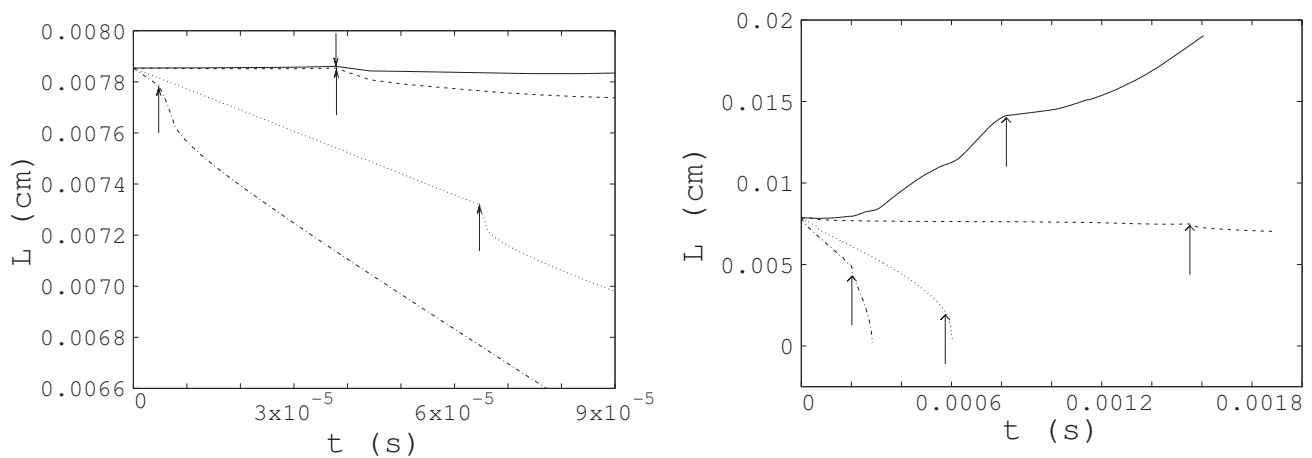

FiguRE 7. Comparison of vortex ring length evolution for the initial ring radius $R=0.00125 \mathrm{~cm}$ case. Lines are: $T=0 \mathrm{~K}$ (solid), $T=1.3 \mathrm{~K}$ (dashed), $T=1.95 \mathrm{~K}$ (dotted) and $T=2.171 \mathrm{~K}$ (dot-dashed). Left: vortex-ring length for small times. The arrows mark the points where the loop attaches to the particle. Right: vortex-ring length for all computation times. The arrows mark the points where the loop detaches from the particle.

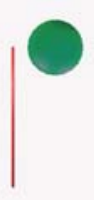

(a)

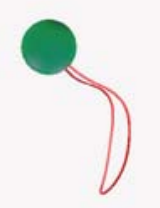

(d)

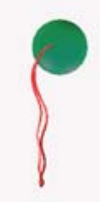

(b)

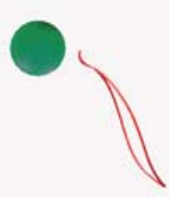

(e)

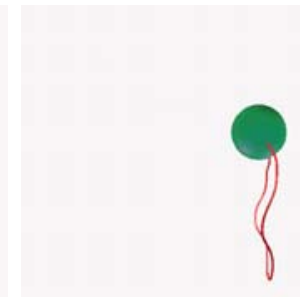

(c)

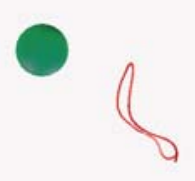

(f)

Figure 8. (Colour online.) Particle-vortex collision for initial particle speed $V=0 \mathrm{~cm} \mathrm{~s}^{-1}$, at $T=1.3 \mathrm{~K}$. The vortex-ring has radius $R=0.00025 \mathrm{~cm}$ and moves to the right with initial velocity $3.4346 \mathrm{~cm} \mathrm{~s}^{-1}$. The initial vortex-ring plane is normal to the figure. Time increases from the upper-left graph towards the lower-right graph as follows: (a) $t_{1}=0 \mathrm{~s}$, (b) $t_{2}=0.2524 \times 10^{-4} \mathrm{~s}$, (c) $t_{3}=0.5048 \times 10^{-4} \mathrm{~s}$, (d) $t_{4}=0.1073 \times 10^{-3} \mathrm{~s}$, (e) $t_{5}=0.1514 \times 10^{-3} \mathrm{~s}$, (f) $t_{6}=0.1704 \times 10^{-3} \mathrm{~s}$. The trajectory of the loop is similar to the corresponding $T=0 \mathrm{~K}$ case (figure 2). However, there are differences, such as for example the mutual friction-induced loop smoothness.

between particle-vortex collision and separation. After detachement, in contrast to the $T=0 \mathrm{~K}$ case, the particle dynamics is determined mainly by the viscous drag force, and the particle is brought to a halt.

Figure 10 shows the system's configuration for the $R=0.00125 \mathrm{~cm}$ case. Mutual friction damps the collision-induced Kelvin waves, and, in a sense, the vortex is tethered at its upper end by the particle. Since the lower vortex part is not strongly affected by the col- 

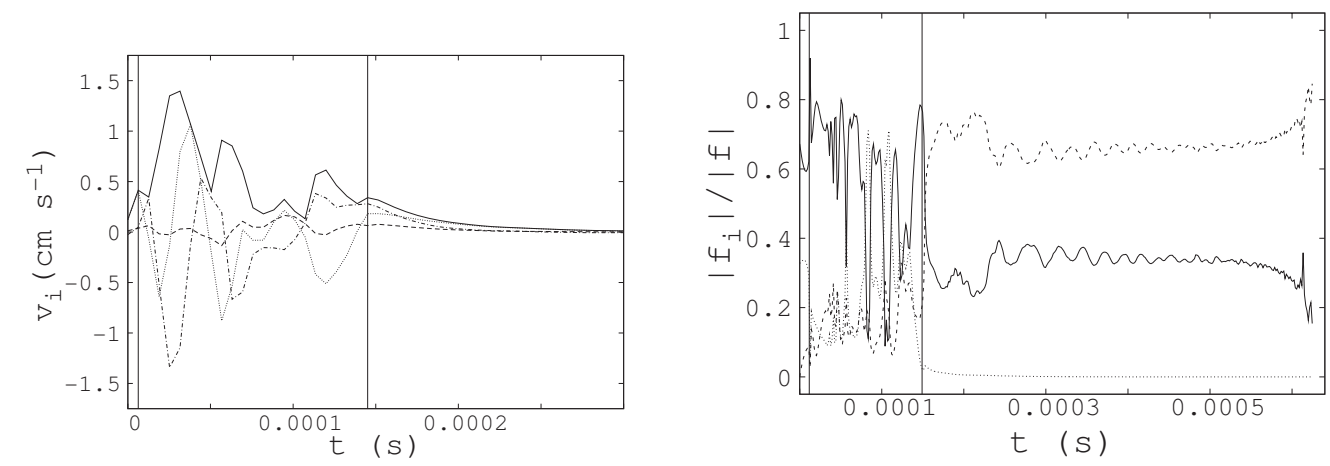

Figure 9. Particle-vortex collision for initial particle speed $V=0 \mathrm{~cm} \mathrm{~s}^{-1}$, at $T=1.3 \mathrm{~K}$. The particle's diameter is $2 a=2 \times 10^{-4} \mathrm{~cm}$, the ring radius is $R=0.00025 \mathrm{~cm}$, and the initial particle-vortex distance is $1.5 a$. The solid vertical lines mark the time of particle-vortex collision and separation. Left: particle velocity evolution. The dashed line signifies the $x$-component, the dot-dashed line corresponds to the $y$-component, and the $z$-component is depicted by the dotted line. The solid line indicates the total velocity magnitude. Even after loop detachement, in contrast to the $T=0 \mathrm{~K}$ case, there are some residual velocity dynamics, since the Stokes drag tends to reduce the particle velocity to zero. Right: partial contributions to the total force magnitude. As long as the vortex is attached to the sphere, the most dominant forces are the local (solid line) and boundary force (dotted line); after loop-particle separation, the drag force (dashed line) dominates, and reduces the particle velocity.

lision, it continues its motion creating a stretched bridge that connects the vortex with the particle. When this bridge becomes too narrow, the vortex reconnects with itself and separates from the particle.

Figure 11 shows the evolutions of velocity (left) and partial force contributions (right). One notes that, as expected, the velocity magnitudes are smaller than those of the $T=0 \mathrm{~K}$ case. The force data suggest that the decisive factor for the dynamics is the development and evolution of Kelvin waves upon particle-vortex collision. Indeed, when $T=0 \mathrm{~K}$, these are allowed to evolve and lead to a large local force which is mainly responsible for the vortex trapping potential. When $T=1.3 \mathrm{~K}$, mutual friction damps these waves and the dynamics are dominated by the boundary force. This state of balance between the forces however, leads to very different system evolutions.

Figure 7 shows the vortex length evolution (dashed lines). Although the collision happens almost at the same time as the collision in the $T=0 \mathrm{~K}$ case, vortex wave damping leads to a much smaller (and in fact monotonically decreasing) vortex length, as well as to a longer period of particle-vortex contact. The rate of length reduction is small, and so the $T=1.3 \mathrm{~K}$ case is an important boundary between the length increasing $T=0 \mathrm{~K}$ evolutions, and the predominantly length decreasing higher temperature cases that we are going to examine next.

\subsection{Temperature $T=1.95 \mathrm{~K}$}

Figure 12 shows the particle-vortex configuration. As shown in figure 7 (dotted lines) the vortex collides with the particle at a later time than in the $T=1.3 \mathrm{~K}$ case (dashed lines). This at first seems to be a paradox, since the mutual friction-induced increased rate of ring-radius reduction would lead one to expect that the vortex would move faster than in the $T=1.3 \mathrm{~K}$ case, and collide with the particle at earlier times. However, one also needs to take into account that, since the particle moves on a steady horizontal plane, the tip of the vortex will shrink below this plane and the vortex needs to cover a larger distance 
(a)

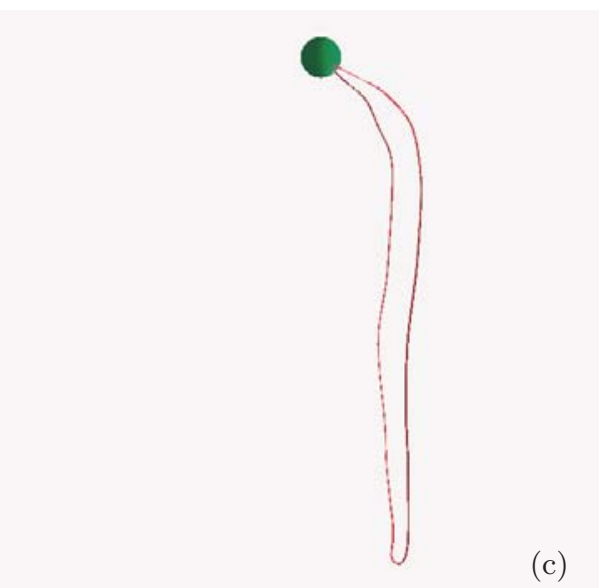

(c)

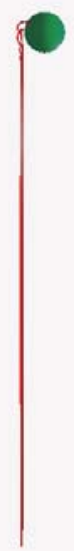

(b)

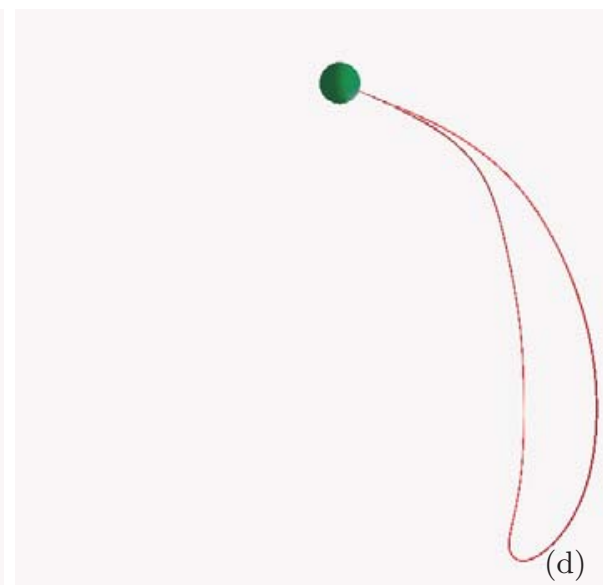

Figure 10. (Colour online.) Particle-vortex collision for initial particle speed $V=0 \mathrm{~cm} \mathrm{~s}^{-1}$, at $T=1.3 \mathrm{~K}$. The vortex-ring has radius $R=0.00125 \mathrm{~cm}$ and moves to the right with initial velocity $0.7868 \mathrm{~cm} \mathrm{~s}^{-1}$. The initial vortex-ring plane is normal to the figure. Time increases from the upper-left graph towards the lower-right graph as follows: (a) $t_{1}=0 \mathrm{~s}$, (b) $t_{2}=0.4417 \times 10^{-4} \mathrm{~s}$, (c) $t_{3}=0.5931 \times 10^{-3} \mathrm{~s}$, (d) $t_{4}=0.1317 \times 10^{-2} \mathrm{~s}$. In opposition to the $T=1.3 \mathrm{~K}, R=0.00025 \mathrm{~cm}$ case, the system's phenomenology here is very different from the corresponding $T=0 \mathrm{~K}$ case. The loop remains smooth and its lower part is not much affected by the collision. As a result, a stretched bridge is created between the main loop and the particle. Evidently, mutual friction, by smoothing out the Kelvin waves on the vortex, neutralizes the trapping effects of the local force, and in conjunction with the viscous drag, damps the spiraling particle trajectory of the corresponding $T=0 \mathrm{~K}$ case.

before collision [figure 12(b)]. This effect is depicted in the results of figure 7 (dotted lines). After collision, the mutual friction causes the vortex to shrink rapidly while still remaining attached to the particle [figure 12(c)], before detaching from it [figure 12(d)]. In order to study the effect of initial particle velocity on the system's dynamics, we have performed a couple of separate computations for the $T=1.95 \mathrm{~K}, R=0.00125 \mathrm{~cm}$ case with initial particle speeds $V=-10 \mathrm{~cm} \mathrm{~s}^{-1}$ and $V=-25 \mathrm{~cm} \mathrm{~s}^{-1}$. The loop length results for these cases (including the $T=2.171 \mathrm{~K}, V=0 \mathrm{~cm} \mathrm{~s}^{-1}$ data for comparison) are shown in figure 13. The results show that, as the initial velocity is increased in the $T=1.95 \mathrm{~K}$ case, the particle attaches to the sphere much earlier than for the $T=2.171 \mathrm{~K}$, $V=0 \mathrm{~cm} \mathrm{~s}^{-1}$ case. Moreover, although for $V=-10 \mathrm{~cm} \mathrm{~s}^{-1}$ the initial velocity effect is 

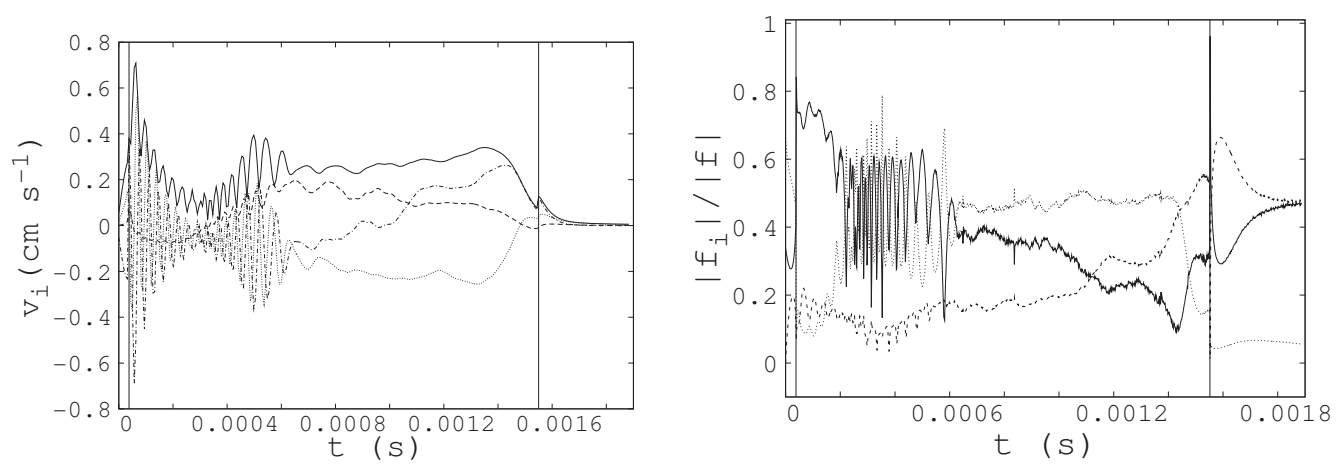

Figure 11. Particle-vortex collision for initial particle speed $V=0 \mathrm{~cm} \mathrm{~s}^{-1}$, at $T=1.3 \mathrm{~K}$. The particle's diameter is $2 a=2 \times 10^{-4} \mathrm{~cm}$, the ring radius is $R=0.00125 \mathrm{~cm}$, and the initial particle-vortex distance is $1.5 \mathrm{a}$. The solid vertical lines mark the times of particle-vortex collision and separation. Left: particle velocity evolution. The shaking of the particle by the vortex is illustrated by the oscillations of the velocity along the $y-$ (dot-dashed line) and $z$ - directions (dotted line). The solid line signifies the total velocity magnitude, and the dashed line is the $x$-component of velocity. Right: partial contributions to the total force magnitude. The local force (solid line) is dominant immediately after the collision. Its importance decays with time, as the mutual friction smooths the vortex contour, and the boundary force (dotted line) becomes the dominant one. Viscous drag (dashed line) has a small influence on the dynamics in between the collision and separation times, but becomes eventually the dominant force.

not strong enough to differentiate strongly the vortex length dynamics from their zero initial velocity counterparts, there is an obvious effect for $V=-25 \mathrm{~cm} \mathrm{~s}^{-1}$. For this case, figure 14 indicates that, throughout the system's evolution, the lower part of the vortex is unaffected by its collision with the particle, and only its upper part is stretched by the particle upon detachement. This stretching is responsible for the increase of vortex length observed in figure 13. Overall, the ratio between particle-vortex contact time-scale and mutual friction action time-scale is an important dynamical factor at these high temperatures.

The results for the particle velocity and the particle forces are shown in figure 15 . Figure 15 (right) indicates that, the superfluid induced forces dominate the dynamics throughout the period of particle-vortex contact. The normal-fluid induced viscous drag becomes important only around the onset of detachement. This is due to the fact that the ring has shrank drastically by the time of detachment, and in fact dissappears completely immediately after. The latter explains why the superfluid induced forces drop sharply to zero at $t=0.000604 \mathrm{~s}$. Both the $y$ - and $z$-components of the particle velocity become negative before the onset of collision [figure 15 (left)]. This behaviour is explained by noticing that the vortex initially moves with its velocity pointing toward the positive $y$-axis, and at the same time (as shown in figure 12), shrinks toward the negative $z$-axis. Thus, the particle, since it is attracted by the vortex and tends to move towards it, develops negative velocity components along the $y-$ and $z$-axes. Upon collision, a low pressure develops in the area of particle vortex contact, and the resulting force rapidly pushes the particle along the negative $y$-axis. However, the viscous drag force, which is strong around collision time, strives to bring the particle to a halt, and so the post-collisional $y$-velocity tends subsequently towards positive values. In accord with these, notice that a plateau in the graph of the $y$-velocity is associated with domination of the viscous force by the superfluid induced forces, and that further non-trivial dynamics are possible only towards detachement, when the balance of forces is adjusted 
(a)

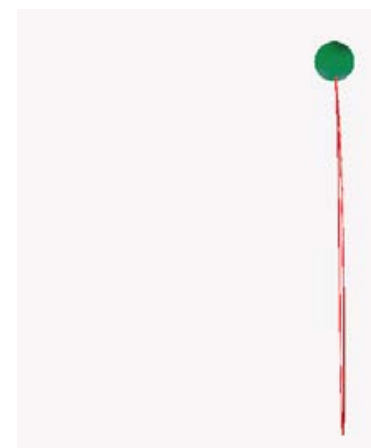

(c)

(d)

Figure 12. (Colour online.) Particle-vortex collision for initial particle speed $V=0 \mathrm{~cm} \mathrm{~s}^{-1}$, at $T=1.95 \mathrm{~K}$. The vortex-ring has radius $R=0.00125 \mathrm{~cm}$ and moves to the right with initial velocity $0.2725 \mathrm{~cm} \mathrm{~s}^{-1}$. The initial vortex-ring plane is normal to the figure. Time increases from the upper-left graph towards the lower-right graph as follows: (a) $t_{1}=0 \mathrm{~s}$, (b) $t_{2}=0.6941 \times 10^{-4} \mathrm{~s}$, (c) $t_{3}=0.3723 \times 10^{-3} \mathrm{~s}$, (d) $t_{4}=0.5805 \times 10^{-3} \mathrm{~s}$. The particle shrinks much faster than in the $T=1.3 \mathrm{~K}$ case, and so it has to travel a longer distance before it collides with the particle [figure 12(b)]. While attached to the particle [figure 12(c)], the vortex shrinks significantly. Since the upper vortex part is tethered by the particle, it tends to be stretched by the lower part that continues its forward motion. This effect eventually causes particle-vortex detachement [figure 12(d)] by inducing a self-reconnection between the segments of the stretched bridge.

once again.

\subsection{Temperature $T=2.171 \mathrm{~K}$}

Figure 16 shows the time evolution of the system's configuration. One notes that according to figure 7 (dot-dashed lines), the particle-vortex collision [figure 16(b)] happens at an earlier time than for the $T=1.95 \mathrm{~K}$ case (dotted lines). This is explained by the fact that, due to enhanced mutual friction action at this high temperature, the ring-radius shrinks at a dramatic rate and accelerates accordingly. Moreover (figure 7), it is remarkable that despite the higher temperature, at the time of detachment [figure 16(d)], the ring has shrank less than at the corresponding $T=1.95 \mathrm{~K}$ case separation time. This 

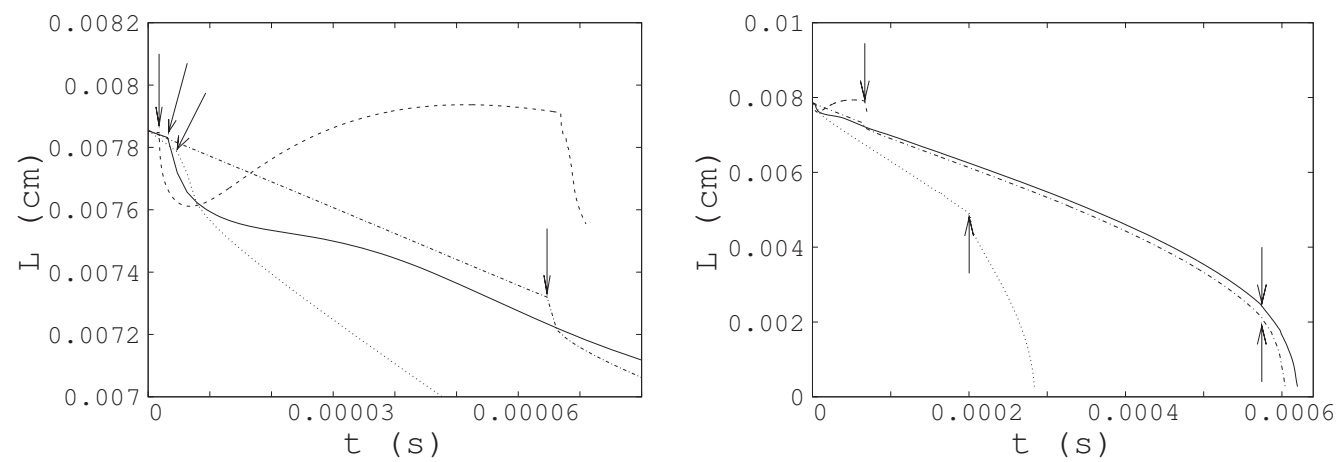

Figure 13. Comparison of initial vortex ring length evolution for radius $R=0.00125 \mathrm{~cm}$. Lines are: $T=1.95 \mathrm{~K}, V=0 \mathrm{~cm} \mathrm{~s}^{-1}$ (dot-dashed), $T=1.95 \mathrm{~K}, V=-10 \mathrm{~cm} \mathrm{~s}^{-1}$ (solid), $T=1.95 \mathrm{~K}, V=-25 \mathrm{~cm} \mathrm{~s}^{-1}$ (dashed), and $T=2.171 \mathrm{~K}, V=0 \mathrm{~cm} \mathrm{~s}^{-1}$ (dotted). Left: vortex-ring length for small times. The arrows mark the points where the loop attaches to the particle. Right: vortex-ring length for all computation times. The arrows mark the points where the loop detaches from the particle.

is because, due to its higher velocity (because of higher shrinking rate) relative to the $T=1.95 \mathrm{~K}$ case, the ring does not stay in contact with the particle for a long time, and so mutual friction does not have enough time to act.

For this case too, we have performed a series of computations in order to investigate the effects of initial particle velocity. It was found that, as the initial velocity increases, the vortex is vigorously deformed as it tries to avoid the approaching particle. This process is active at all temperatures, but it is more pronounced at $T=2.171 \mathrm{~K}$ due to higher values of the mutual friction coefficients. Figure 17 demonstrates the deformation mechanism for initial velocity $V=-1000 \mathrm{~cm} \mathrm{~s}^{-1}$. As the vortex and the particle approach each other [figure 17(a)], the vortex is deformed into an arc that tends to avoid the particle [figure 17(b) and figure 17(c)]. However, at the points where the arc meets the (relatively) undisturbed part of the vortex, the vortex curvature is high and increases proportionally to the growth of the arc radius. At the points of this increased curvature, the local vortex velocity becomes very high, and so the vortex rapidly collapses on to the particle as indicated by the arrows of figure $17(\mathrm{~d})$. After the collision [figure 17(e)], the particle acquires a vortex handle [figure $17(\mathrm{f})$ ]. This intriguing phenomenon begs the question of whether there is a threshold velocity above which the vortex avoids the particle. In order to answer this question, we have gradually increased the initial particle velocity. We found that even up to speed values $V=-10000 \mathrm{~cm} \mathrm{~s}^{-1}$ the vortex always collides with the particle.

The results for the particle forces and particle velocities for speed $V=0 \mathrm{~cm} \mathrm{~s}^{-1}$ are shown in figure 18. Figure 18 (left) suggests that upon collision, the particle is sharply pushed along the leftward-downward direction. This forcing is due to the pressure difference between the low pressure area formed by vortex-particle contact and the pressure on the upper hemisphere of the particle. Subsequently, this perturbation is strongly damped and there are no particle oscillations. The increased mutual friction effects reduce the impact of the local force immediately after the collision, and Stokes damping becomes the most significant particle force. Notice the differences with the $T=1.95 \mathrm{~K}$ case (figure 15). They are due to aforementioned differences in the location (along the particle surface) and timing of the collision in the two cases. Due to the particular vortex-particle configuration shown in figure 16(c), a low pressure area is induced on the right particle hemisphere close to particle-vortex detachement. The resulting pressure difference 


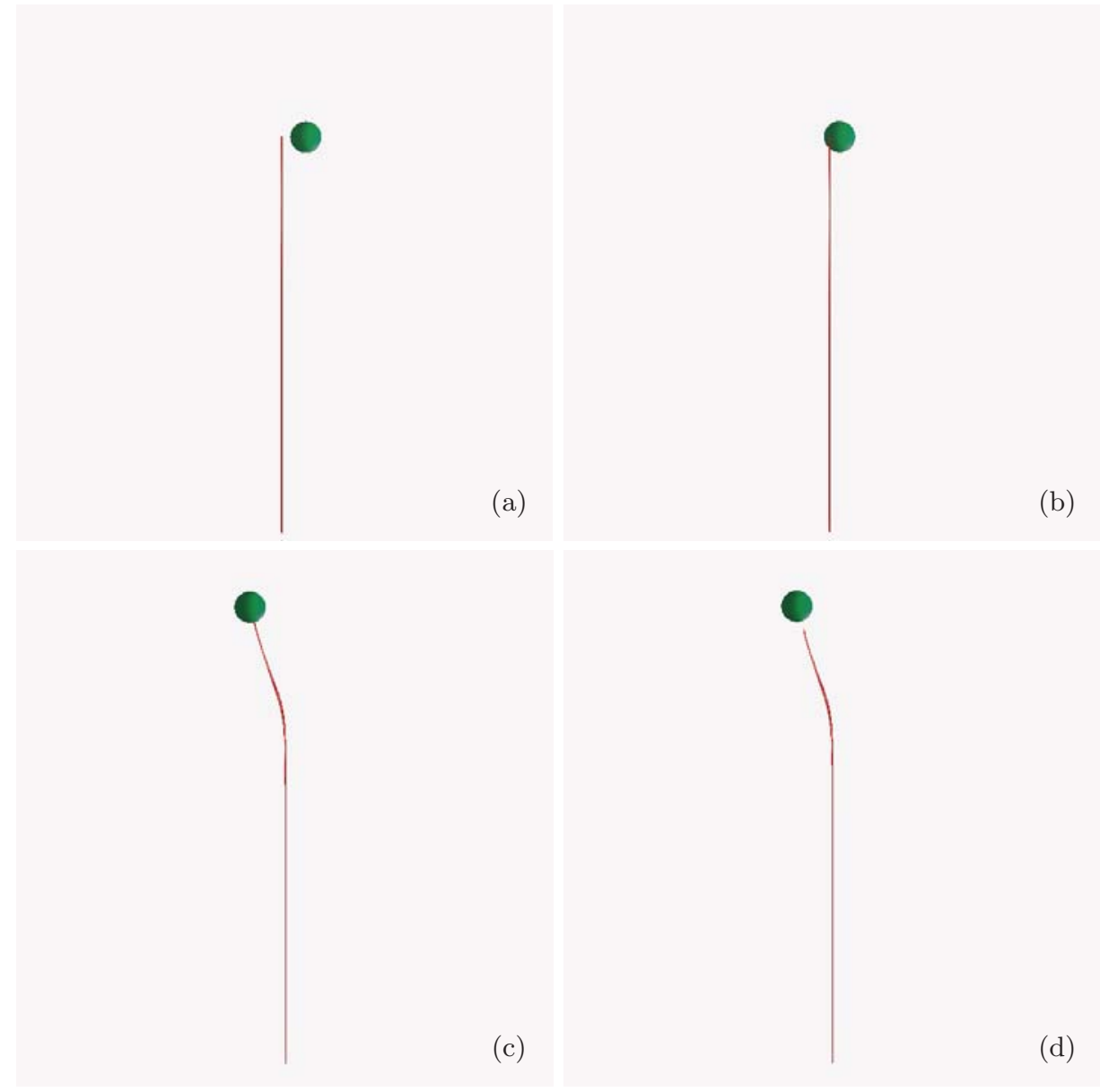

Figure 14. (Colour online.) Particle-vortex collision for initial particle speed $V=-25 \mathrm{~cm} \mathrm{~s}^{-1}$, at $T=1.95 \mathrm{~K}$. The vortex-ring has radius $R=0.00125 \mathrm{~cm}$ and moves to the right with initial velocity $0.3047 \mathrm{~cm} \mathrm{~s}^{-1}$. The initial vortex-ring plane is normal to the figure. Time increases from the upper-left graph towards the lower-right graph as follows: (a) $t_{1}=0 \mathrm{~s}$, (b) $t_{2}=0.3786 \times 10^{-5} \mathrm{~s}$, (c) $t_{3}=0.6562 \times 10^{-4} \mathrm{~s}$, (d) $t_{4}=0.6814 \times 10^{-4} \mathrm{~s}$. The effect of initial velocity becomes clear when we compare with the results of figure 12 . The ring spends a small amount of time in contact with the particle, and has scarcely shrank upon detachement.

is responsible for the observed positive particle velocity component along the $y$-direction.

\section{Conclusion}

We have performed self-consistent computations of the interactions between a superfluid vortex ring and a solid-particle for two different ring sizes and over a wide range of temperatures starting from absolute zero and ending close to the superfluid transition temperature. At non-zero temperatures, we have kinematically prescribed a motionless normal-fluid component. The ratio of vortex-ring radius over the particle radius was $R / a=2.5$ for the smaller ring case, and $R / a=12.5$ for the larger ring case. We have found that, in all cases, the particle and the vortex eventually separate.

For $T=0 \mathrm{~K}$, larger rings tend to trap the particles more effectively than smaller rings. 

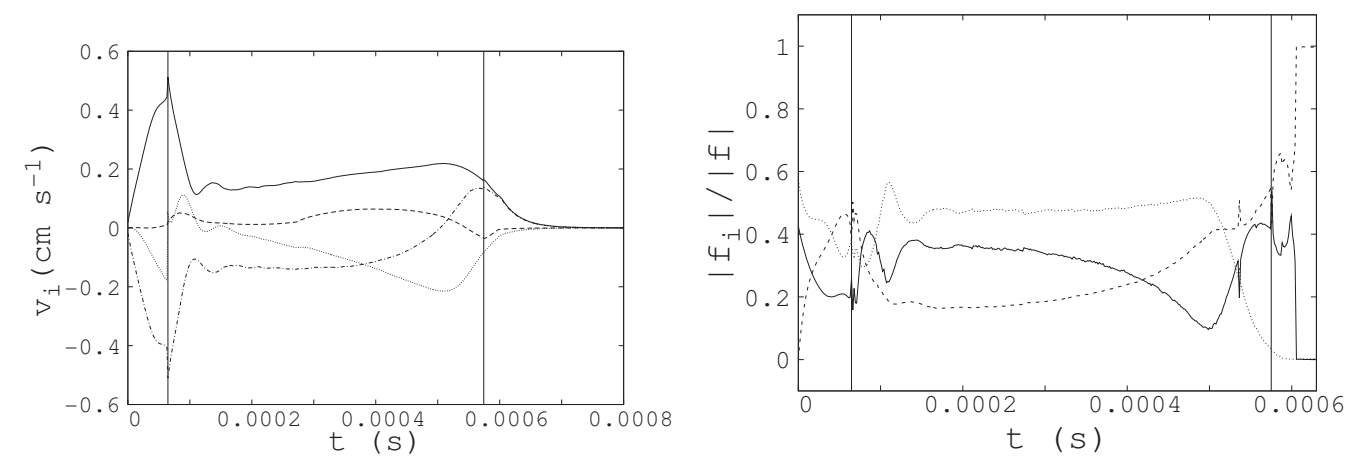

FiguRE 15. Particle-vortex collision for initial particle speed $V=0 \mathrm{~cm} \mathrm{~s}^{-1}$, at $T=1.95 \mathrm{~K}$. The particle's diameter is $2 a=2 \times 10^{-4} \mathrm{~cm}$, the ring radius is $R=0.00125 \mathrm{~cm}$, and the initial particle-vortex distance is $1.5 a$. The solid vertical lines mark the times of particle-vortex collision and separation. Left: particle velocity evolution. The dashed line signifies the $x$-component, the dot-dashed line corresponds to the $y$-component, and the $z$-component is depicted by the dotted line. The solid line indicates the total velocity magnitude. The particle is sharply pushed along the negative $y$-axis upon collision, and the effects of this forcing are subsequently damped by viscous drag effects. Right: partial contributions to the total force magnitude. The lines signify the local force (solid), the boundary induced force (dotted) and the viscous drag force (dashed). The superfluid induced forces dominate the dynamics throughout the period of particle-vortex contact.

Due to the absence of mutual friction effects, the rings are vigorously distorted by the collision induced Kelvin waves, and loose their ring-like appearance. The $T=0 \mathrm{~K}$ results also suggest that an experiment monitoring the motion of solid-particles in a superfluid vortex link could detect particle-vortex interactions by recording the spiral-like particle motions depicted in figure 4 . The larger the rings involved in the process are, the more evident the particle motions would be, since larger rings are more effective than smaller ones in their efforts to trap particles. As indicated by the data, the larger ring induces much higher post-collisional particle velocities than the smaller one. Moreover, due to the undamped collision induced vortex wave system, the local force is grossly overpowering the boundary force.

For $T>0 \mathrm{~K}$, the particle vibrates normal to its direction of motion upon its collision with the vortex. The solutions suggest that smaller rings induce larger vibration velocities. This is exactly opposite to what happens in the $T=0 \mathrm{~K}$ case, and suggests that, for $T>0 \mathrm{~K}$, smaller rings might be easier to detect experimentally. At smaller temperatures (here $T=1.3 \mathrm{~K}$ and $T=1.95 \mathrm{~K}$ ), the superfluid induced forces dominate the normal-fluid induced viscous damping between collision and detachement. As the temperature increases, the increased mutual friction damping of vortex waves (that are the main contributors to the local force) leads to the damping of both the intensity and frequency of post-collision particle vibrations. Overall, as the temperature increases, the importance of the local force is reduced and that of the drag force is increased. This also helps to damp the particle oscillations. In fact, at $T=2.171 \mathrm{~K}$, the viscous force is the largest contributor to particle dynamics between collision and detachement. With the exception of $T=0 \mathrm{~K}$, the boundary force is always an important dynamical factor. According to the $T=1.3 \mathrm{~K}$ data, the collision of particles with vortices would result in sudden particle vibrations (as indicated by the particle velocity data) that could be detected if the vortex system is dilute enough for the particles to move relatively smoothly (i.e. without fluctuations) in between their collisions with the superfluid vortices. The latter is conditioned upon the type and energy level of the corresponding normal-fluid 
(a)

(c)
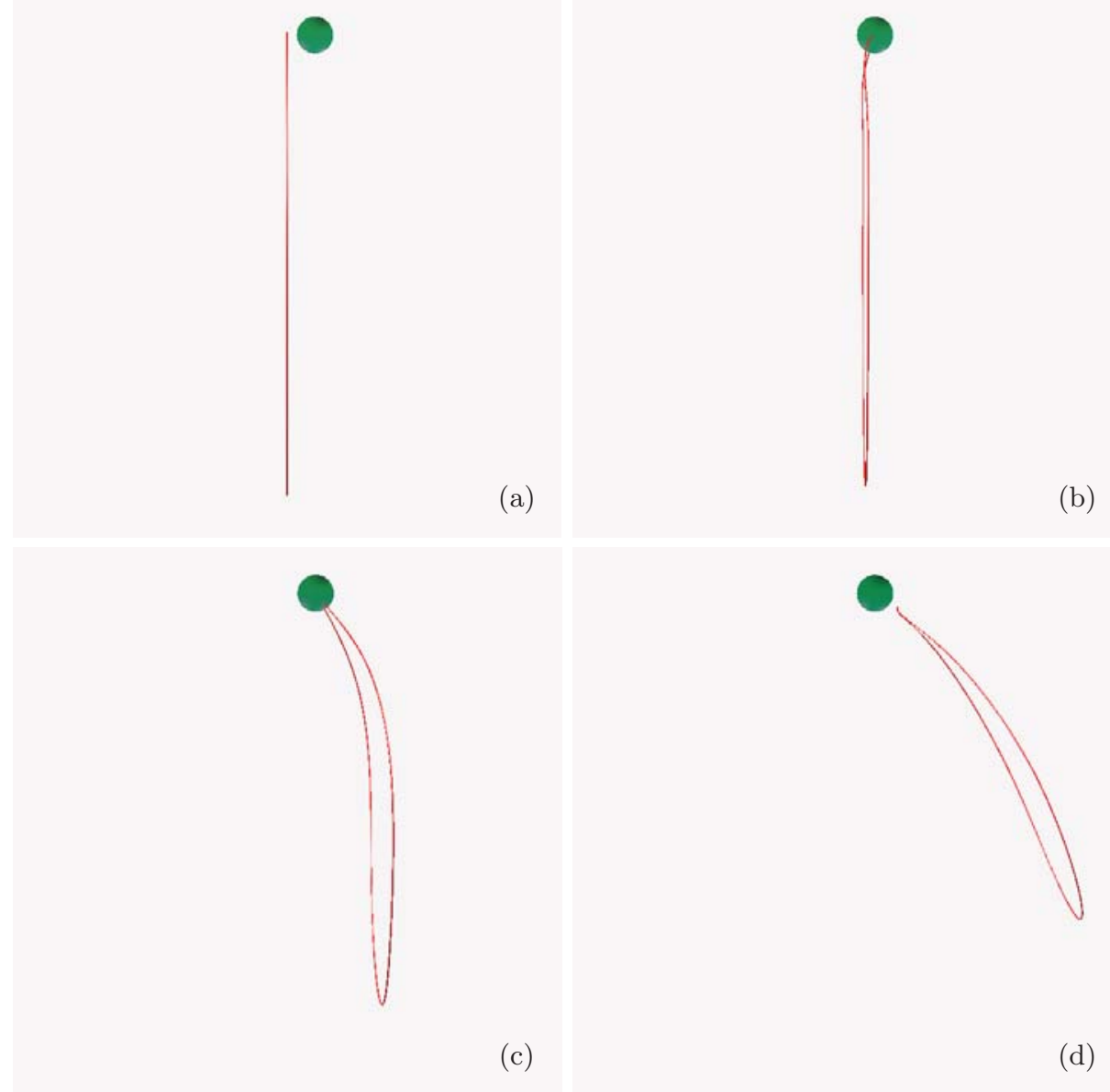

(b)

Figure 16. (Colour online.) Particle-vortex collision for initial particle speed $V=0 \mathrm{~cm} \mathrm{~s}^{-1}$, at $T=2.171 \mathrm{~K}$. The vortex-ring has radius $R=0.00125 \mathrm{~cm}$ and moves to the right with initial velocity $4.7225 \mathrm{~cm} \mathrm{~s}^{-1}$. The initial vortex-ring plane is normal to the figure. Time increases from the upper-left graph towards the lower-right graph as follows: (a) $t_{1}=0 \mathrm{~s}$, (b) $t_{2}=0.1893 \times 10^{-4} \mathrm{~s}$, (c) $t_{3}=0.9622 \times 10^{-4} \mathrm{~s}$, (d) $t_{4}=0.2033 \times 10^{-3} \mathrm{~s}$.

flow. One could imagine, for example, a turbulent normal-fluid component. For this case, Kivotides et al. (2007a) showed that a solid-particle would most of the time sharply change its direction upon encountering a normal-fluid filament, but, sporadically, also briefly spiral around it. These motions are different from the normal to the particle's direction of motion vibrations depicted here, so, in principle, the effects of the two fluids on the particles could be discernible. In the case of turbulent normal-fluid, an interesting possibility has to do with the presence of the coherent superfluid bundles calculated in Kivotides (2006). In particular, according to the present results, when a superfluid vortex arc that is curved at the scale of the particle collides with the latter, the two will separate after their interaction. In contrast, for vortices that appear as straight lines at the scale of a particle, Kivotides et al. (2008) have shown that, for each $T>0 \mathrm{~K}$, there is a corresponding range of incoming particle velocities for which particle and vortex stay together after collision. With respect to this, note also that Kivotides (2005) has shown that, for $T=1.3 \mathrm{~K}$, a tangle of superfluid vortices interacting with (a stationary on the average) normal-fluid presents a scaling regime in the histogram of the local vortex curvature, 


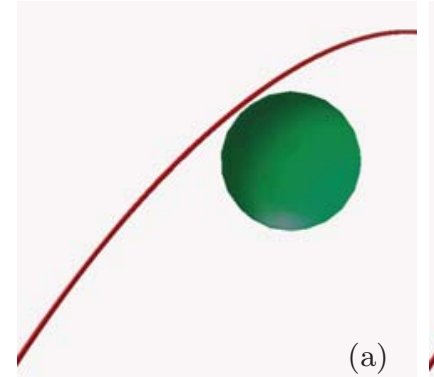

(a)

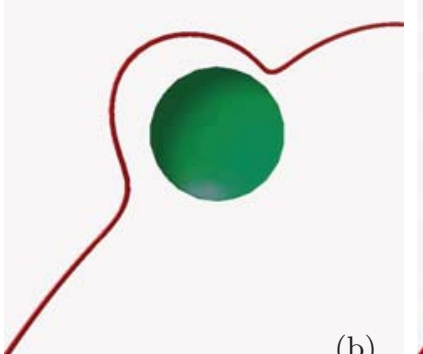

(b)

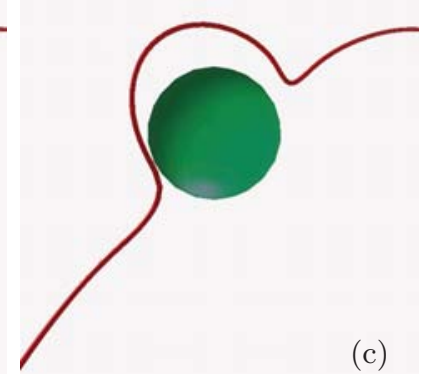

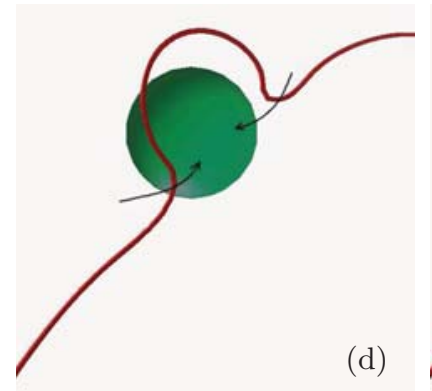
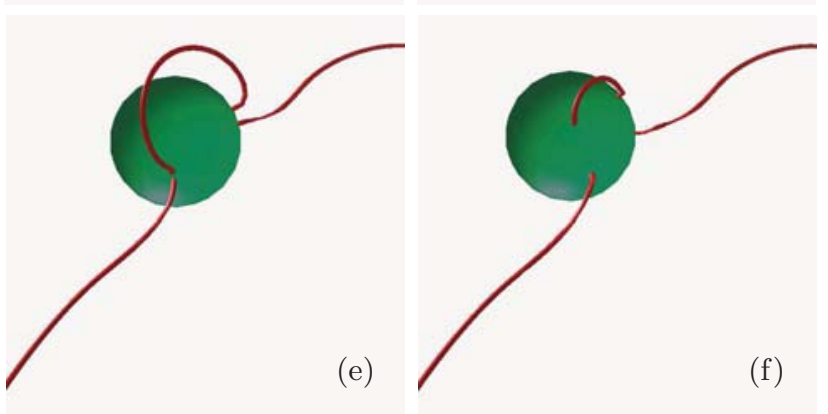

Figure 17. (Colour online.) Particle-vortex collision for initial particle speed $V=-1000 \mathrm{~cm} \mathrm{~s}^{-1}$, at $T=2.171 \mathrm{~K}$. The vortex-ring has radius $R=0.00125 \mathrm{~cm}$ and moves to the right with initial velocity $42.3787 \mathrm{~cm} \mathrm{~s}^{-1}$. For clarity, only part of the vortex loop is shown. Time increases from the upper-left graph towards the lower-right graph as follows: (a) $t_{1}=0 \mathrm{~s}$, (b) $t_{2}=0.1059 \times 10^{-6} \mathrm{~s}$, (c) $t_{3}=0.1414 \times 10^{-6} \mathrm{~s}$, (d) $t_{4}=0.1750 \times 10^{-6} \mathrm{~s}$, (e) $t_{5}=0.1888 \times 10^{-6} \mathrm{~s}$, (f) $t_{6}=0.2226 \times 10^{-6} \mathrm{~s}$. As the particle-ring approach deforms the latter, the high curvature loop segments formed at the roots of the induced vortex arc cause the loop to collapse upon the particle forming a handle.

and that the cut-off of this regime is $R_{c} \approx 10^{-5} \mathrm{~m}$. This curvature radius coincides with the radius of the larger ring computed here. In this context, Kivotides (2006) has shown that, in turbulent liquid helium, the normal-fluid might induce stretched vortex-bundles of macroscopic circulation in the superfluid. When a solid-particle collides with such a bundle of vortices, it might be trapped by them by having its kinetic energy radiated from the point of collision via vortex waves propagating along the bundle. This in turn, in combination with the results of Kivotides (2007), suggests that the induced Kelvin waves, for sufficiently high particle kinetic energy, could excite bundle instability modes that would lead to the destruction of the superfluid coherent structures. In order to address such crucial issues, a combined action of experiment and theory is needed.

It is important to note that our computations assume that the particle is a perfect sphere, hence they ignore any surface roughness at the vortex core scale. There are two reasons behind this assumption. First, the mathematical method that computes the effect of the particle-boundary on the vortices is valid only when the former is a perfect sphere. This is because the spherical shape allows the derivation of an analytical formula for the effect of the boundary on a linear vortex segment which is one of the main results upon which the present computations are based. Second, the introduction of small scale roughness requires a similar refinement of the discretization length along the vortices which is not computationally feasible. The employed grid is already computationally demanding, and it only resolves adequately the sphere-radius scale phenomena. Having said these, we do not expect the qualitative conclusions of this study to be altered by the inclusion of finescale dynamics. Indeed, the eventual particle-vortex detachement is due to the large-scale 

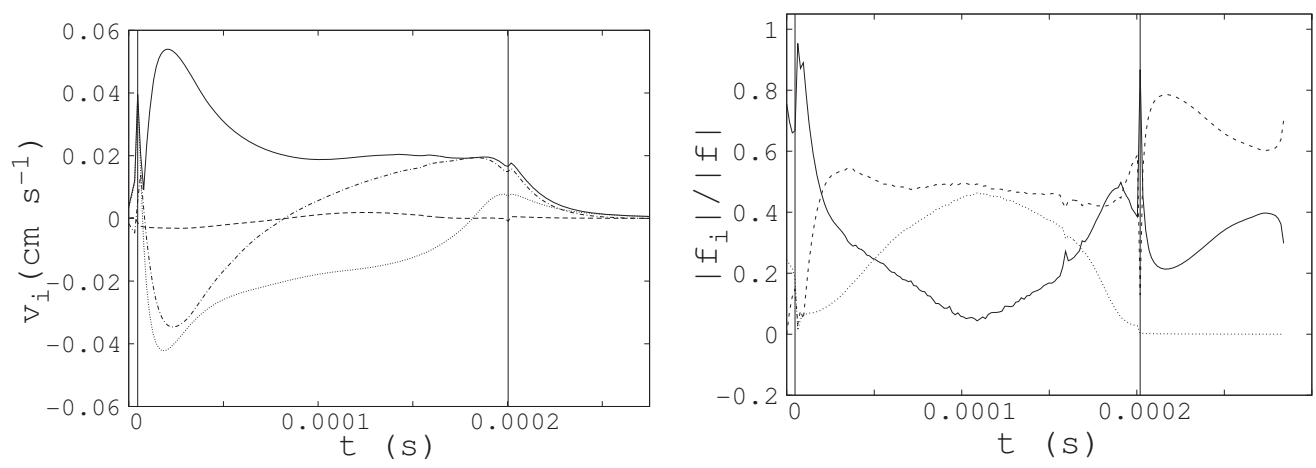

Figure 18. Particle-vortex collision for initial particle speed $V=0 \mathrm{~cm} \mathrm{~s}^{-1}$, at $T=2.171 \mathrm{~K}$. The particle's diameter is $2 a=2 \times 10^{-4} \mathrm{~cm}$, the ring radius is $R=0.00125 \mathrm{~cm}$, and the initial particle-vortex distance is $1.5 a$. The solid vertical lines mark the times of particle-vortex collision and separation. Left: particle velocity evolution. The dashed line signifies the $x$-component, the dot-dashed line corresponds to the $y$-component, and the $z$-component is depicted by the dotted line. The solid line indicates the total velocity magnitude. After a strong push in the downward-leftward direction upon collision, viscous drag action damps the particle's motion. Close to detachement, the $y$-velocity acquires positive values. This is due to the particular vortex-particle configuration of figure 16(c), that induces a low pressure area on the right particle hemisphere pushing the particle to the right. Right: partial contributions to the total force magnitude. The lines signify the local force (solid), the boundary induced force (dotted) and the viscous drag force (dashed). During most of the particle-vortex contact period, the particle dynamics are governed by the competition between boundary and viscous forces.

velocity of the ring relative to the particle which is determined by the initial ring-radius. Moreover, the relative importance of the contributions of the various forces to particle dynamics depends mostly on the establishment upon collision of a (well resolved at the particle-radius scale) Kelvin wave system on the vortices, and not on the wavenumberspace extension of the spectrum of this system. Finally, another important assumption is that of a kinematically prescribed stationary normal-fluid velocity field. This assumption could be relaxed by developing methods for the calculation of self-consistent interactions between the three fields comprising multiphase liquid helium physics: superfluid, normalfluid, and particulate-solid. This requires (in addition to the methods employed here), the computation of the normal-fluid flow around a suspension of spherical particles. At present, we can only speculate about the physics of such complex interactions. It is well known (Kivotides, Barenghi \& Samuels 2000), that a superfluid vortex ring moving in an initially at rest normal-fluid generates two normal-fluid vortices around it. Thus, when the superfluid vortex ring collides with the particle, it is as if two normal-fluid vortices also collide with the latter. Moreover, the normal flow around the particle would deform the vortex-ring contour even before collision. For small Reynolds number, creeping normal-flow around the particle, the above effects might not be very important, but for higher particle velocities there could be a significant effect that deserves systematic study. Overall, the future development of fully dynamic, self-consistent methods would allow a lively interaction between experiment and theory in liquid helium, which could help advance our understanding of these remarkable systems. 
Barenghi, C. F., Donnelly, R. J. \& Vinen, W. F. 2001 Quantized Vortex Dynamics And Superfluid Turbulence. Springer, Berlin.

Barenghi, C. F., Donnelly, R. J. \& Vinen, W. F. 1983 Friction on quantized vortices in He II . A review. J. Low Temp. Phys. 52, 189.

Bewley, G. P., Lathrop, D. P. \& Sreenivasan, K. R. 2006 Superfluid helium: visualization of quantized vortices. Nature 44,588.

Brennen, C. E. 2005 Fundamentals of Multiphase Flow. Cambridge University Press.

Celik, D. \& VanSciver, S. W. 2002 Tracer particle generation in superfluid helium through cryogenic liquid injection for particle image velocimetry (PIV) applications. Exp. Thermal and Fluid Sci. 26, 971-975.

Crowe, C. T., Sommerfeld, M. S. \& Tsuji, Y. 1998 Multiphase Flows with Droplets and Particles. CRC Press, Boca Raton.

DeWaele, A. T. A. M. \& Aarts, R. G. K. M. 1994 Route to vortex reconnection. Phys. Rev. Lett. 72, 482.

Donnelly, R. J. 1991 Quantised Vortices In Helium II. Cambridge University Press.

Donnelly, R. J., Karpetis, A. N., Niemela, J. J., Sreenivasan, K. R., Vinen, W. F. \& White, C. M. 2002 The use of particle image velocimetry in the study of turbulence in liquid helium. J. Low Temp. Phys. 126, 327-332.

Drew, D. A. \& Passman, S. L. 1998 Theory of Multicomponent Fluids. Springer, Berlin.

Finne, A. P., Eltsov, V. B., Hanninen, R., Kopnin, N. B., Kopu, J., Krusius, M., TsubOTA, M. \& Volovik, G. E. 2006 Dynamics of vortices and interfaces in superfluid ${ }^{3} \mathrm{He}$. Rep. Prog. Phys. 69, 3157-3230.

Kivotides, D. 2005 Turbulence without inertia in thermally excited superfluids. Phys. Lett. A 341, 193-197.

Kivotides, D. 2006 Coherent structure formation in turbulent thermal superfluids. Phys. Rev. Lett. 96, 175301.

Kivotides, D. 2007 Relaxation of superfluid vortex bundles via energy transfer to the normal fluid. Phys. Rev. B 76, 054503.

Kivotides, D., Barenghi, C. F., Mee, A. J. \& Sergeev, Y. A. 2007 a Interaction of solid particles with a tangle of vortex filaments in a viscous fluid. Phys. Rev. Lett. 99, 074501.

Kivotides, D., Barenghi, C. F. \& Samuels, D. C. 2000 Triple vortex ring structure in superfluid helium II. Science 290, 777.

Kivotides, D., Barenghi, C. F. \& Sergeev, Y. A. 2005 Measurement of the normal fluid velocity in superfluids. Phys. Rev. Lett. 95, 215302.

Kivotides, D., Barenghi, C. F. \& Sergeev, Y. A. 2006 a Numerical calculation of the interaction of superfluid vortices and a rigid sphere. J. Low Temp. Phys. 144, 121-134.

Kivotides, D., Barenghi, C. F. \& Sergeev, Y. A. $2006 b$ Physics of solid particles in thermal counterflow. Europhys. Lett. 73, 733-739.

Kivotides, D., Barenghi, C. F. \& Sergeev, Y. A. $2007 b$ Collision of a tracer particle and a quantized vortex in superfluid helium: Self-consistent calculations. Phys. Rev. B 75, 212502.

Kivotides, D., Barenghi, C. F. \& Sergeev, Y. A. 2008 Interactions between particles and quantized vortices in superfluid helium. Phys. Rev. B 77, 014527.

Kivotides, D., Vassilicos, J. C., Samuels, D. C. \& Barenghi, C. F. 2001 Kelvin waves cascade in superfluid turbulence. Phys. Rev. Lett. 86, 3080.

Kozik, E. \& Svistunov, B. 2004 Scale-Separation Scheme for Simulating Superfluid Turbulence: Kelvin-Wave Cascade. Phys. Rev. Lett. 92, 035301.

Nazarenko, S. \& West, R. J. 2003 Analytical Solution for Nonlinear Schroedinger Vortex Reconnection. J. Low Temp. Phys. 132, 1.

Poole, D. R., Barenghi, C. F., Sergeev, Y. A. \& Vinen, W. F. 2005 The motion of tracer particles in helium II. Phys. Rev. B 71, 064514.

Schwarz, K. W. 1974 Spherical probes and quantized vortices: Hydrodynamic formalism and simple applications. Phys. Rev. A 10, 2306.

Schwarz, K. W. 1985 Three-dimensional vortex dynamics in superfluid ${ }^{4}$ He: Line-line and line-boundary interactions. Phys. Rev. B 31, 5782.

Tsubota, M. \& Maekawa, S. 1993 Pinning and depinning of two quantized vortices in superfluid ${ }^{4}$ He. Phys. Rev. B 47, 12040. 
Vinen, W. F. \& Niemela, J. J. 2002 Quantum turbulence. J. Low Temp. Phys. 129, 213.

Vinen, W. F., Tsubota, M., \& Mitani, A. 2003 Kelvin-Wave Cascade on a Vortex in Superfluid 4 He at a Very Low Temperature. Phys. Rev. Lett. 91, 135301.

Winckelmans, G. S. \& Leonard, A. 1993 Contributions to vortex particle methods for the computation of three-dimensional incompressible unsteady flows. J. Comput. Phys. 109, 247.

Zhang, T., Celik, D. \& VanSciver, S. W. 2004 Tracer particles for applications to PIV studies of liquid helium. J. Low Temp. Phys. 134, 985-1000.

Zhang, T. \& VAnSciver, S. W. 2005 Large-scale turbulent flow around a cylinder in counterflow superfluid $4 \mathrm{He}$ (He (II)). Nature Phys. 1, 36-38. 